\title{
Icephobic, Pt-cured, Polydimethylsiloxane Nanocomposite Coatings
}

\author{
Yongfeng Liu ${ }^{1,2,3}$, Chenyu Wang ${ }^{2}$, Rebecca M. Jarrell, ${ }^{2}$ Sithara Nair, ${ }^{2}$ Kenneth J. Wynne ${ }^{2 *}$ \\ and Duolong $\mathrm{Di}^{1,3}$
}

1. CAS Key Laboratory of Chemistry of Northwestern Plant Resources and Key Laboratory for Natural Medicine of Gansu Province, Lanzhou Institute of Chemical Physics, Chinese Academy of Sciences (CAS), Lanzhou 730000, People’s Republic of China

2. Department of Chemical and Life Science Engineering, School of Engineering, Virginia Commonwealth University, 601 West Main Street, Richmond, Virginia 23284, United States

3. Center of Resource Chemical and New Material, 36 Jinshui Road, Qingdao 266100, People’s Republic of China

$\ddagger$ These authors contributed equally to this work.

Corresponding Author: kjwynne@vcu.edu

Part 1. Leaching, contact angles and ice adhesion. Dynamic contact angles were obtained by the Wilhelmy plate method that involves coating a coverslip and immersion in water. Details are in the Experimental section. The characterization focused on Pt-PDMS(25) and Pt-PDMS(100) MQ-R coatings (1) to obtain contact angles, especially the receding contact angle $\left(\theta_{R}\right)$ which is connected to adhesion of water to silicone coatings and (2) to determine whether or not leaching (oils, surfactants, processing materials) affected the adhesion of ice. Systematic changes in work of adhesion and the connection to adhesion of ice have been presented and discussed in the main paper. In the section below, evidence for leaching is presented. In summary, we were not able to 
connect changes in leaching into water during Wilhelmy plate measurements to ice adhesion.

Table S1 lists DCA data taken for repeated runs on Pt-PDMS(25) and Pt-PDMS(100) MQ-R coatings. Receding contact angles for Pt-PDMS(100) coatings are reasonably stable while variations are observed for coatings prepared at ambient temperature. Figure S2 shows Wilhelmy plate force distance curves (fdc's) using a flamed glass slide for testing water after DCA analysis. ${ }^{1-2}$ If oils or other contaminants such as processing aids leach and rise to the water/air interface the surface tension of water decreases. Evidence for leaching from PtPDMS(25) coatings is reflected in changes for $\theta_{\mathrm{A}}$ and $\theta_{\mathrm{R}}$ from first to third DCA runs for 0 to 30 wt\% MQ-R filled (Table S1). For example, for the first DCA cycle for Pt-PDMS(25)-20 is $131^{\circ}$, which decreases to $119^{\circ}$ for cycle 3 . This decrease of about $10^{\circ}$ is typical for 0 to $30 \mathrm{wt} \% \mathrm{MQ}-\mathrm{R}$ coatings and is apparent from inspection of force distance curves in Figure S2. Interestingly, for $25^{\circ} \mathrm{C}$ cure, only Pt-PDMS(25)-40 is free of water contamination due to leached species which is attributed to an increasingly complex path for diffusing species with increasing MQ-R content.

Minimal water contamination is found for Pt-PDMS(100) coatings. Condensation cure at $100{ }^{\circ} \mathrm{C}$ forms a second network that may provide a contorted pathway for leaching or low molar mass species may be incorporated in the network. In addition, volatile species are simply removed. 
Table S1. Wilhelmy plate DCA advancing and receding CAs for MQ-R filled PtPDMS(25) and Pt-PDMS(100). ${ }^{\mathrm{a}}$

\begin{tabular}{|c|c|c|c|c|c|c|c|c|c|}
\hline \multirow{3}{*}{ MQ-R wt\% } & \multirow{3}{*}{ Cycle } & \multicolumn{4}{|c|}{ Pt-PDMS(25) } & \multicolumn{4}{|c|}{ Pt-PDMS(100) } \\
\hline & & \multicolumn{2}{|c|}{ Run 1} & \multicolumn{2}{|c|}{ Run 2} & \multicolumn{2}{|c|}{ Run 1} & \multicolumn{2}{|c|}{ Run 2} \\
\hline & & $\theta_{\mathrm{A}}$ & $\theta_{\mathrm{R}}$ & $\theta_{\mathrm{A}}$ & $\theta_{\mathrm{R}}$ & $\theta_{\mathrm{A}}$ & $\theta_{\mathrm{R}}$ & $\theta_{\mathrm{A}}$ & $\theta_{\mathrm{R}}$ \\
\hline \multirow{3}{*}{0} & Cycle 1 & 124 & 60 & 124 & 61 & 117 & 89 & 115 & 84 \\
\hline & Cycle 2 & 117 & 68 & 117 & 69 & 114 & 86 & 112 & 82 \\
\hline & Cycle 3 & 116 & 69 & 117 & 68 & 113 & 94 & 111 & 82 \\
\hline \multirow{3}{*}{5} & Cycle 1 & 136 & 52 & 136 & 40 & 115 & 91 & 116 & 92 \\
\hline & Cycle 2 & 122 & 48 & 126 & 45 & 115 & 98 & 115 & 101 \\
\hline & Cycle 3 & 121 & 49 & 122 & 46 & 108 & 101 & 108 & 100 \\
\hline \multirow{3}{*}{10} & Cycle 1 & 133 & 62 & 131 & 61 & 116 & 93 & 116 & 92 \\
\hline & Cycle 2 & 128 & 60 & 129 & 61 & 116 & 96 & 116 & 91 \\
\hline & Cycle 3 & 124 & 60 & 125 & 61 & 111 & 97 & 116 & 99 \\
\hline \multirow{3}{*}{20} & Cycle 1 & 131 & 37 & 135 & 32 & 118 & 93 & 116 & 94 \\
\hline & Cycle 2 & 122 & 48 & 138 & 44 & 116 & 95 & 116 & 94 \\
\hline & Cycle 3 & 119 & 47 & 120 & 43 & 114 & 99 & 117 & 101 \\
\hline \multirow{3}{*}{30} & Cycle 1 & 135 & 24 & 137 & 22 & 119 & 92 & 118 & 90 \\
\hline & Cycle 2 & 137 & 37 & 137 & 34 & 117 & 91 & 119 & 92 \\
\hline & Cycle 3 & 123 & 36 & 122 & 36 & 117 & 92 & 118 & 91 \\
\hline \multirow{3}{*}{40} & Cycle 1 & 127 & 44 & 126 & 42 & 119 & 90 & 119 & 89 \\
\hline & Cycle 2 & 125 & 42 & 125 & 41 & 120 & 89 & 118 & 89 \\
\hline & Cycle 3 & 125 & 43 & 126 & 42 & 119 & 89 & 117 & 90 \\
\hline
\end{tabular}

a. Run 1 and Run 2 refer to different coated coverslips. Multiple runs were carried out to investigate reproducibility. 
Table S2. Wilhelmy plate DCA $\theta_{\mathrm{A}}, \theta_{\mathrm{R}}$ and $\theta_{\Delta}$ for MQ-R filled PtPDMS(25) and Pt-PDMS(100). ${ }^{\mathrm{a}}$

\begin{tabular}{|c|c|c|c|c|c|c|c|}
\hline \multirow{2}{*}{$\begin{array}{c}\text { MQ resin content } \\
(\mathbf{w t} \%)\end{array}$} & \multirow{2}{*}{ Cycle } & \multicolumn{3}{|c|}{ Pt-PDMS(25) } & \multicolumn{3}{|c|}{ Pt-PDMS(100) } \\
\hline & & $\theta_{\mathrm{A}}$ & $\theta_{\mathrm{R}}$ & $\theta_{\Delta}$ & $\theta_{\text {adv }}$ & $\theta_{\text {rec }}$ & $\theta_{\Delta}$ \\
\hline \multirow{3}{*}{$\mathbf{0}$} & Cycle 1 & 124 & 60 & 64 & 117 & 89 & 28 \\
\hline & Cycle 2 & 117 & 68 & 49 & 114 & 86 & 28 \\
\hline & Cycle 3 & 116 & 69 & 47 & 113 & 94 & 19 \\
\hline \multirow{3}{*}{5} & Cycle 1 & 136 & 52 & 84 & 115 & 91 & 24 \\
\hline & Cycle 2 & 122 & 48 & 74 & 115 & 98 & 17 \\
\hline & Cycle 3 & 121 & 49 & 72 & 108 & 101 & 7 \\
\hline \multirow{3}{*}{10} & Cycle 1 & 133 & 62 & 71 & 116 & 93 & 23 \\
\hline & Cycle 2 & 128 & 60 & 68 & 116 & 96 & 20 \\
\hline & Cycle 3 & 124 & 60 & 64 & 111 & 97 & 14 \\
\hline \multirow{3}{*}{20} & Cycle 1 & 131 & 37 & 94 & 118 & 93 & 25 \\
\hline & Cycle 2 & 122 & 48 & 74 & 116 & 95 & 21 \\
\hline & Cycle 3 & 119 & 47 & 72 & 114 & 99 & 15 \\
\hline \multirow{3}{*}{30} & Cycle 1 & 135 & 24 & 111 & 119 & 92 & 27 \\
\hline & Cycle 2 & 137 & 37 & 100 & 117 & 91 & 26 \\
\hline & Cycle 3 & 123 & 36 & 87 & 117 & 92 & 25 \\
\hline \multirow{3}{*}{40} & Cycle 1 & 127 & 44 & 83 & 119 & 90 & 29 \\
\hline & Cycle 2 & 125 & 42 & 83 & 120 & 89 & 31 \\
\hline & Cycle 3 & 125 & 43 & 82 & 119 & 89 & 30 \\
\hline
\end{tabular}

a. Contact angle hysteresis $=\theta_{\Delta}=\theta_{\mathrm{A}}-\theta_{\mathrm{R}}$

b. Contact angle data are from Table S1, Run 1.

Table S3. Receding contact angles for Pt-PDMS coatings and the ratio of work of adhesion (Eq. 1) for water for samples cured at 25 or $100{ }^{\circ} \mathrm{C}$.

\begin{tabular}{|c|c|c|c|c|c}
\hline $\begin{array}{c}\text { MQ resin } \\
\text { content (\%) }\end{array}$ & $\theta_{\mathrm{R}-25}\left(^{\circ}\right)$ & $1+\cos \theta_{\mathrm{R}-25}$ & $\theta_{\mathrm{R}-100}\left(^{\circ}\right)$ & $1+\cos \theta_{\mathrm{R}-100}$ & $\mathrm{~W}_{\mathrm{a}-25} / \mathrm{W}_{\mathrm{a}-100}$ \\
\hline 0 & 60 & 1.50 & 89 & 1.02 & 1.47 \\
\hline 5 & 52 & 1.62 & 91 & 0.98 & 1.64 \\
\hline 10 & 62 & 1.47 & 93 & 0.95 & 1.55 \\
\hline 20 & 37 & 1.80 & 93 & 0.95 & 1.90 \\
\hline 30 & 24 & 1.91 & 92 & 0.97 & 1.98 \\
\hline 40 & 44 & 1.72 & 90 & 1.00 & 1.72 \\
\hline
\end{tabular}


Table S4. Storage modulus $E^{\prime}$ (MPa) for Pt-PDMS(25) and Pt-PDMS(100) at 10 and $-30{ }^{\circ} \mathrm{C}$.

\begin{tabular}{|c|c|c|c|c|}
\hline \multirow{2}{*}{$\begin{array}{c}\text { MQ resin } \\
\text { content (\%) }\end{array}$} & \multicolumn{3}{|c|}{ Storage Modulus $\boldsymbol{E}^{\prime}(\mathbf{M P a})$} \\
\cline { 2 - 5 } & $\mathbf{2 5}{ }^{\circ} \mathbf{C}$ cure & \multicolumn{2}{|c|}{$\mathbf{1 0 0}{ }^{\circ} \mathbf{C}$ cure } \\
\cline { 2 - 5 } & $\mathbf{- 1 0}{ }^{\circ} \mathbf{C}$ & $\mathbf{- 3 0}{ }^{\circ} \mathbf{C}$ & $-\mathbf{- 1 0}^{\circ} \mathbf{C}$ & $\mathbf{- 3 0}{ }^{\circ} \mathbf{C}$ \\
\hline 0 & 1.18 & 1.05 & 0.94 & 0.87 \\
\hline 5 & 1.05 & 1.08 & 1.24 & 1.16 \\
\hline 10 & 1.3 & 1.22 & 1.89 & 1.74 \\
\hline 20 & 1.71 & 1.69 & 2.76 & 2.65 \\
\hline 30 & 2.93 & 3.08 & 6.93 & 8.3 \\
\hline 40 & 4.15 & 5.85 & 13.1 & 18.4 \\
\hline
\end{tabular}
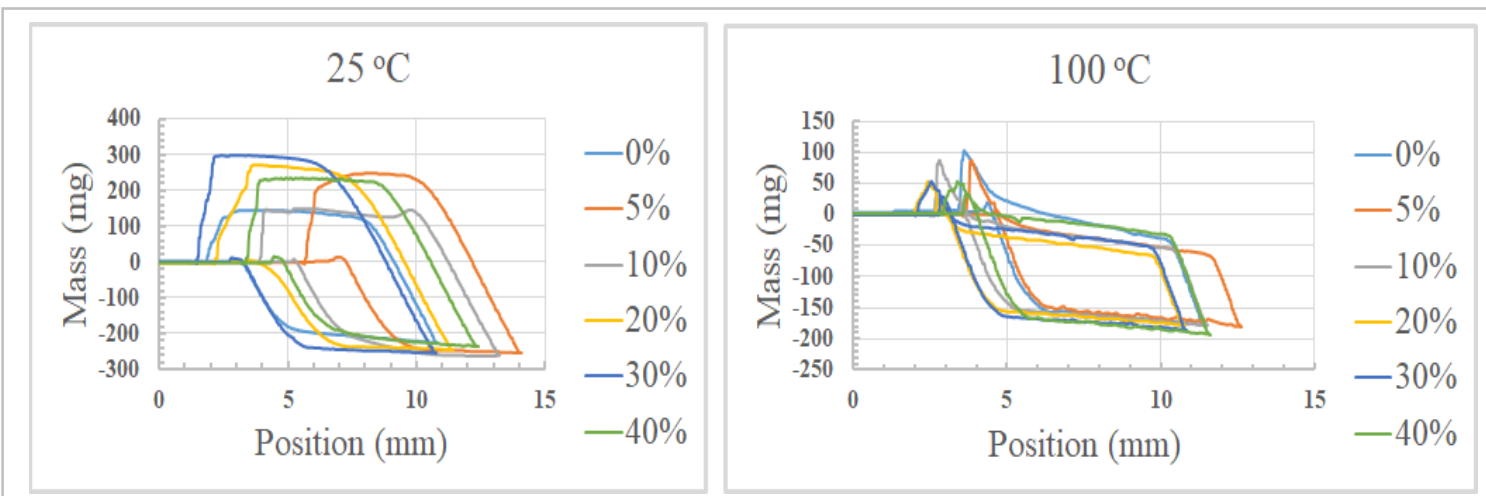

Figure S1. DCA force distance curves for Pt-PDMS coatings. Overall, decreased area for hysteresis loops is evident for $100{ }^{\circ} \mathrm{C}$ cure (right) compared to $25^{\circ} \mathrm{C}$ cure (left). 


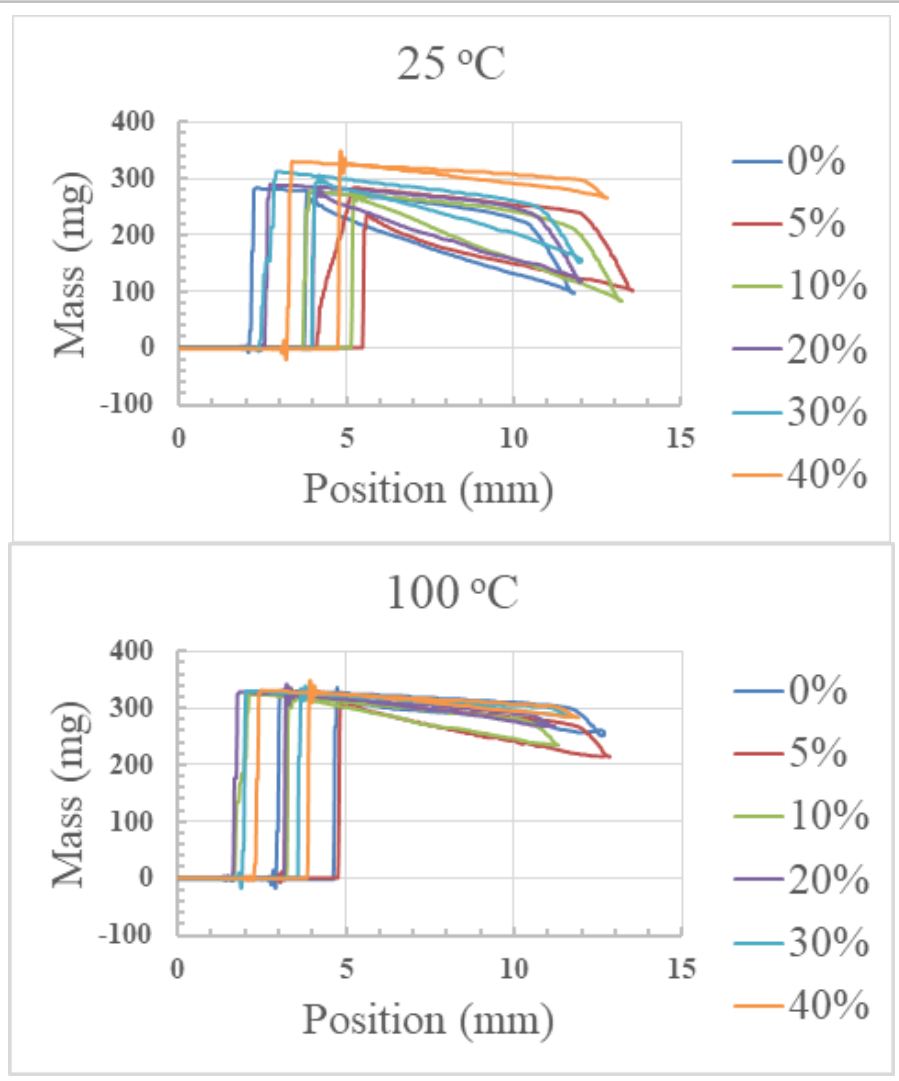

Figure S2. Force distance curves using a flamed glass slide for test water after DCA analysis: top, cure at $25{ }^{\circ} \mathrm{C}$; bottom $100{ }^{\circ} \mathrm{C}$ cure. 


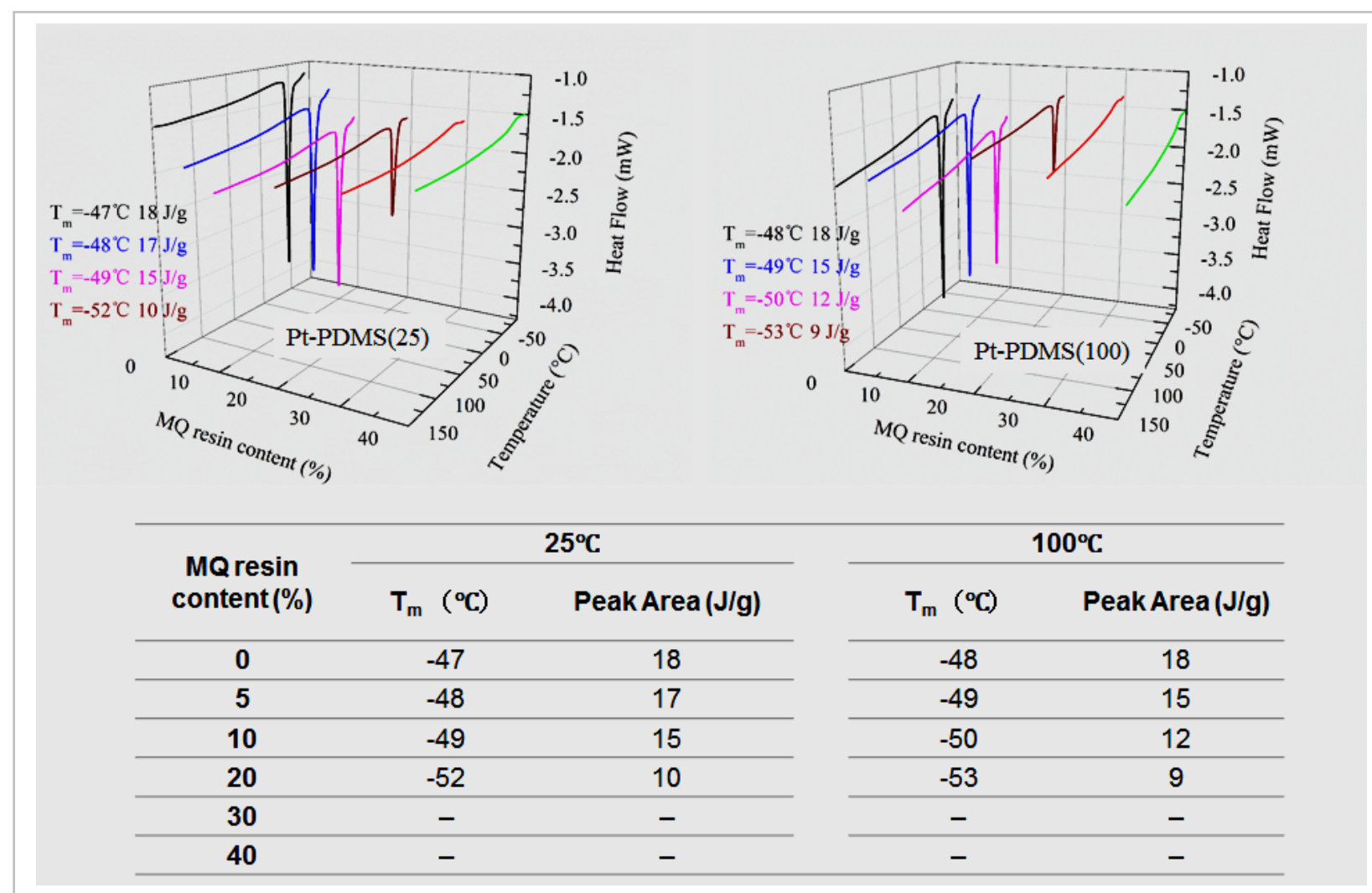

Figure S3. DSC data for Pt-PDMS(25) and Pt-PDMS(100). 


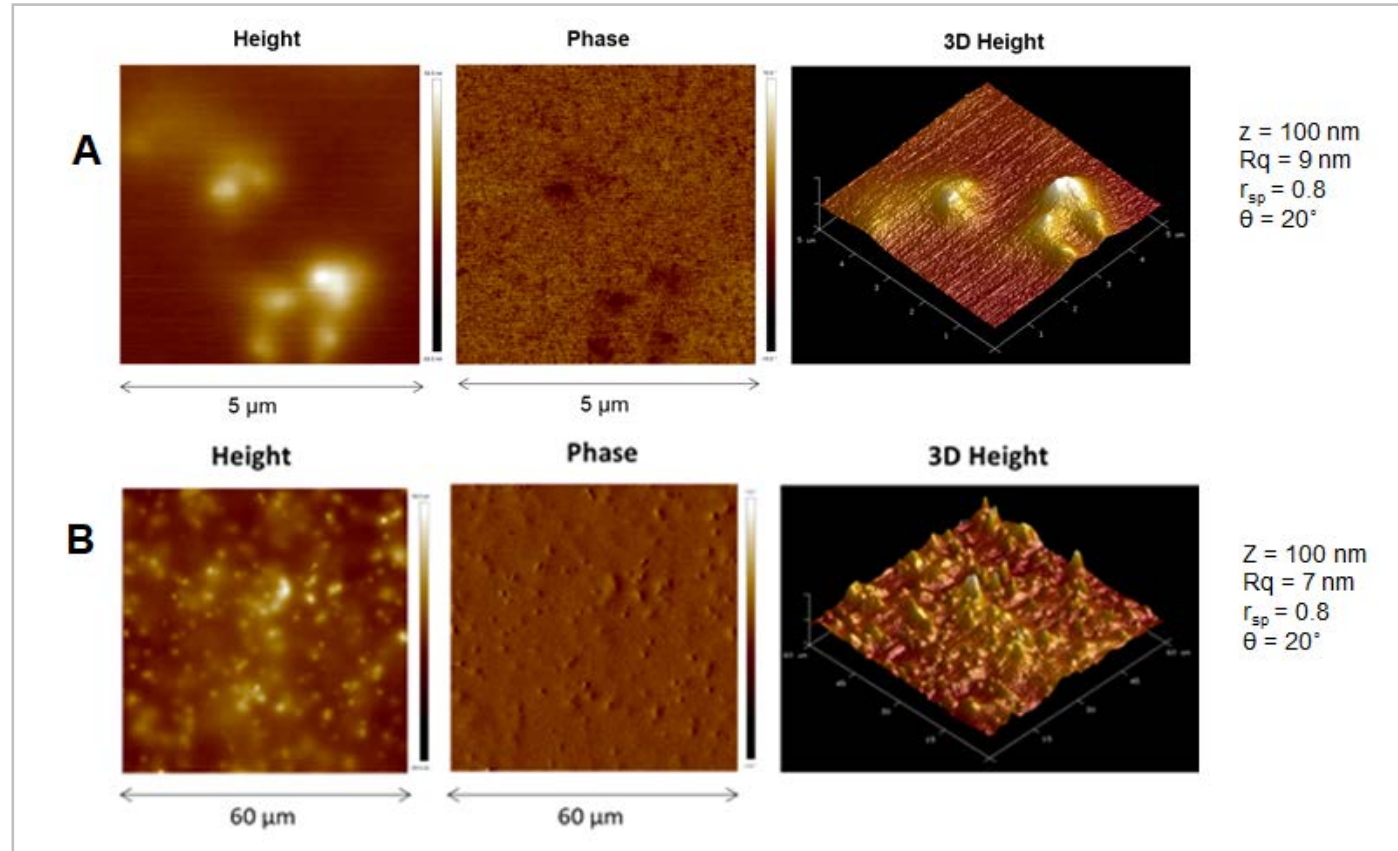

Figure S4. AFM images for Pt-PDMS(25)-30: A, 5 x $5 \mu \mathrm{m}$ and B, 60 x $60 \mu \mathrm{m}$.
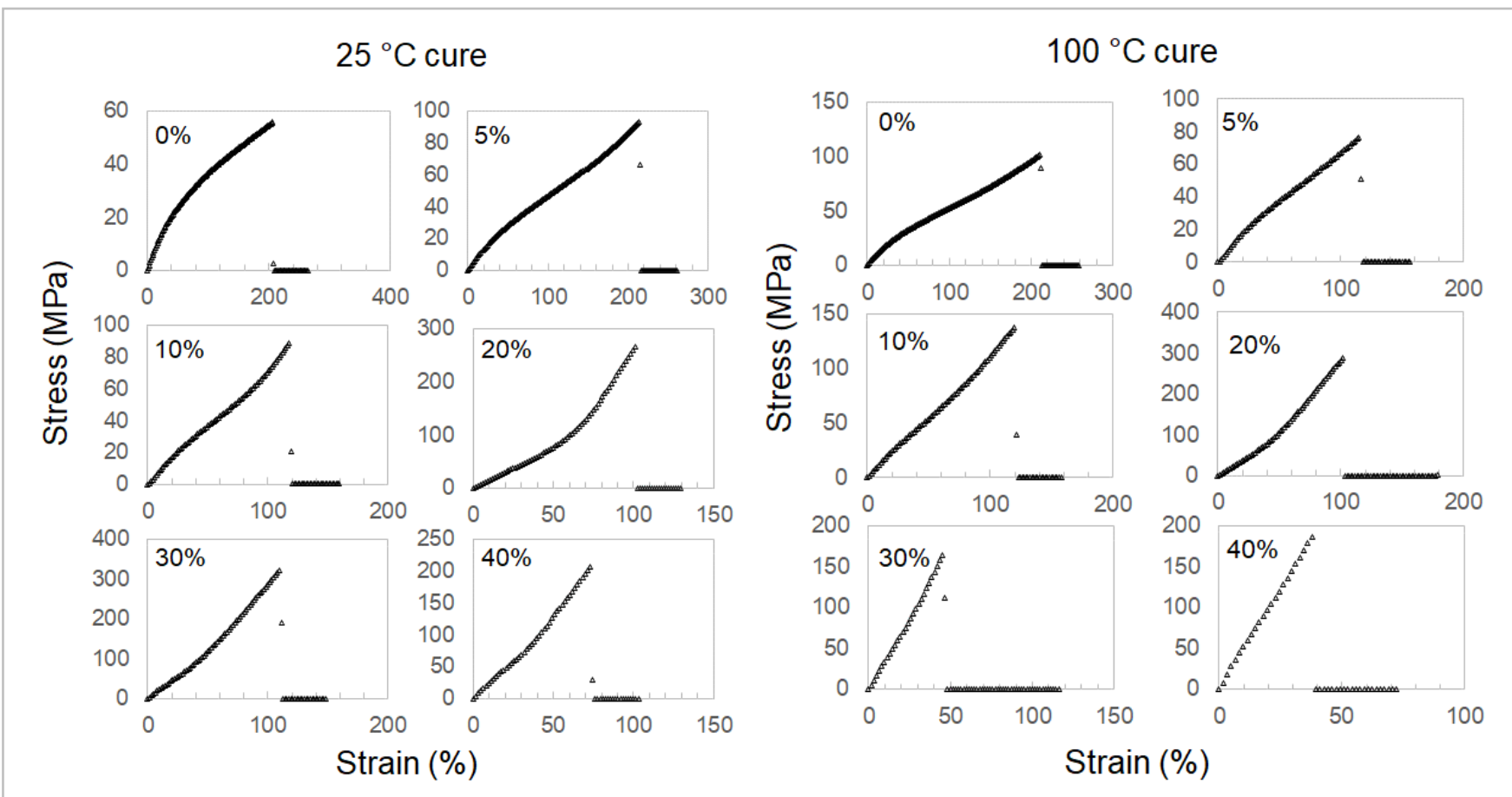

Figure S5. Stress vs strain curves for Pt-PDMS(25) and Pt-PDMS(100) composite coatings. 

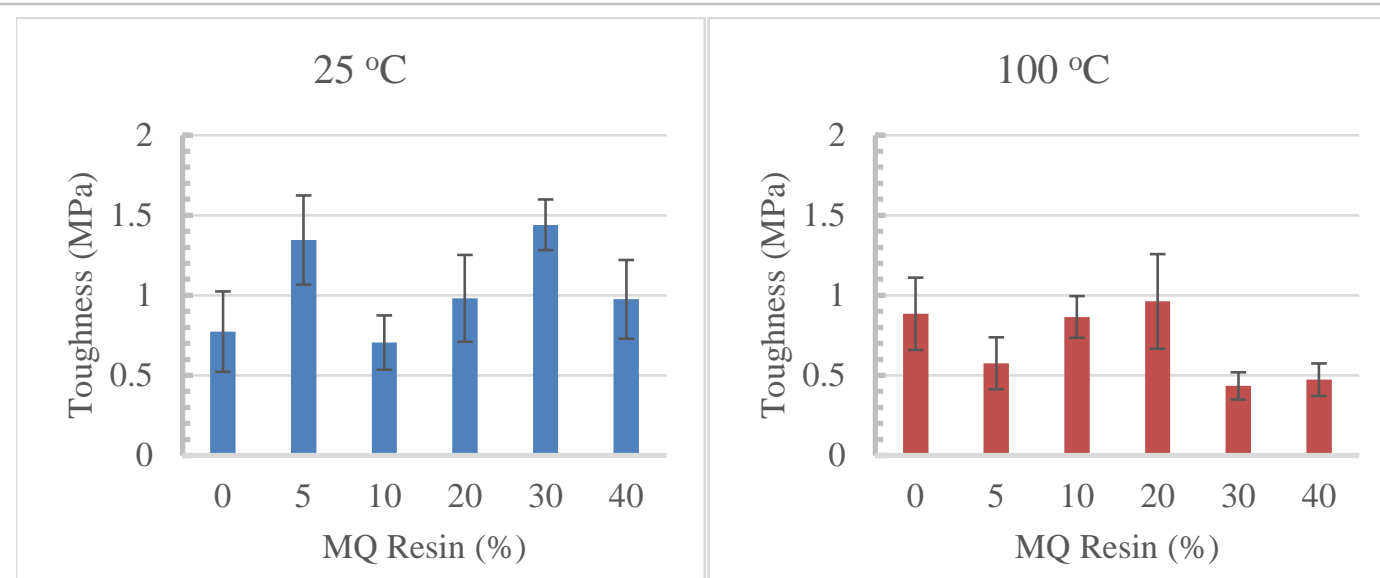

Figure S6. Toughness (area under the stress strain curve) for Pt-PDMS coatings with cured at 25 or $100{ }^{\circ} \mathrm{C}$.

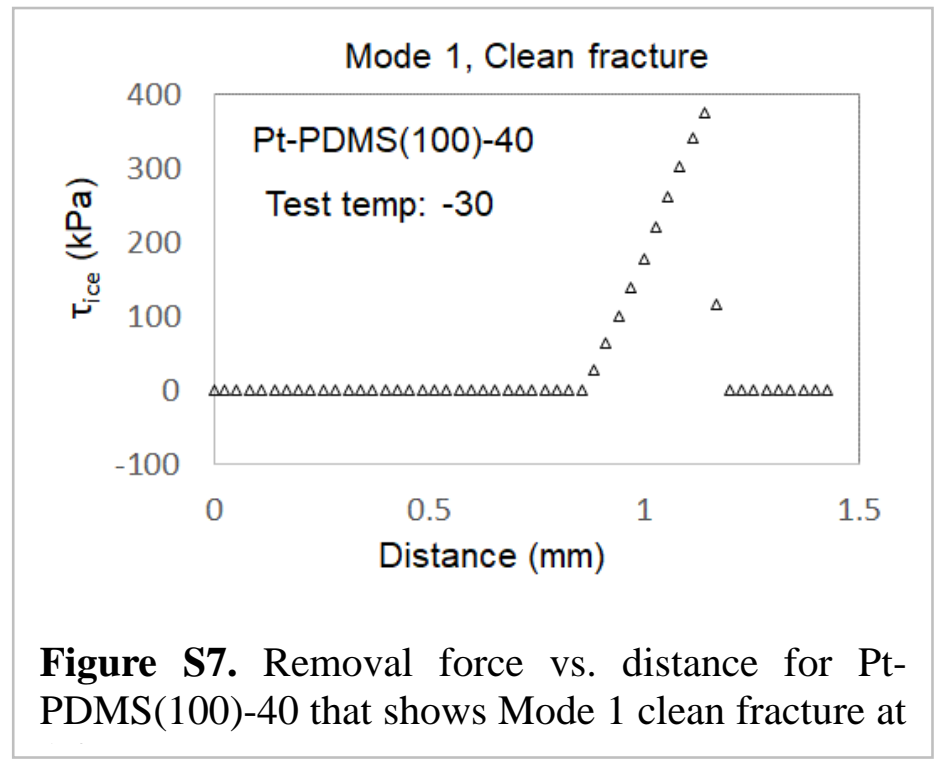




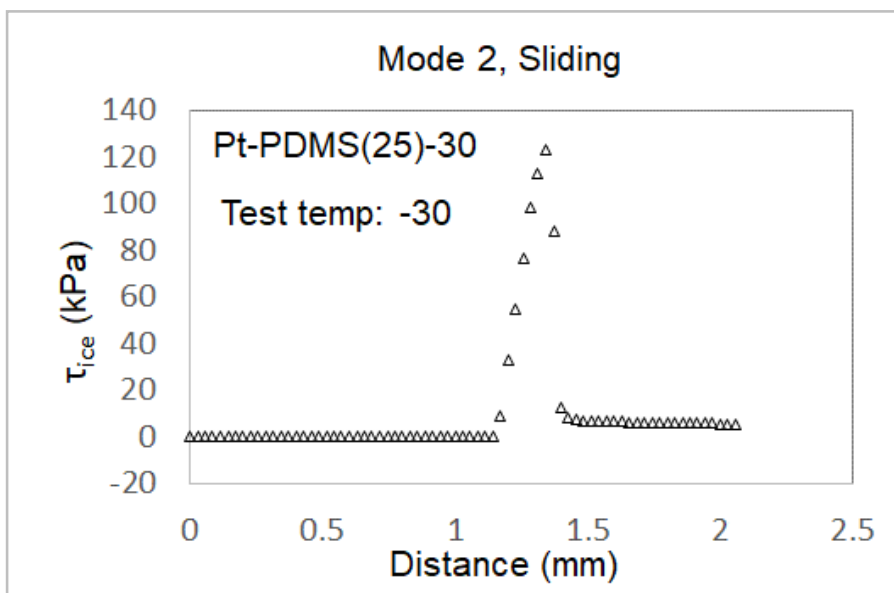

Figure S8. Removal force vs. distance for PtPDMS(25)-30 that shows Mode 2 slippage between 1.3 and $2 \mathrm{~mm}$.

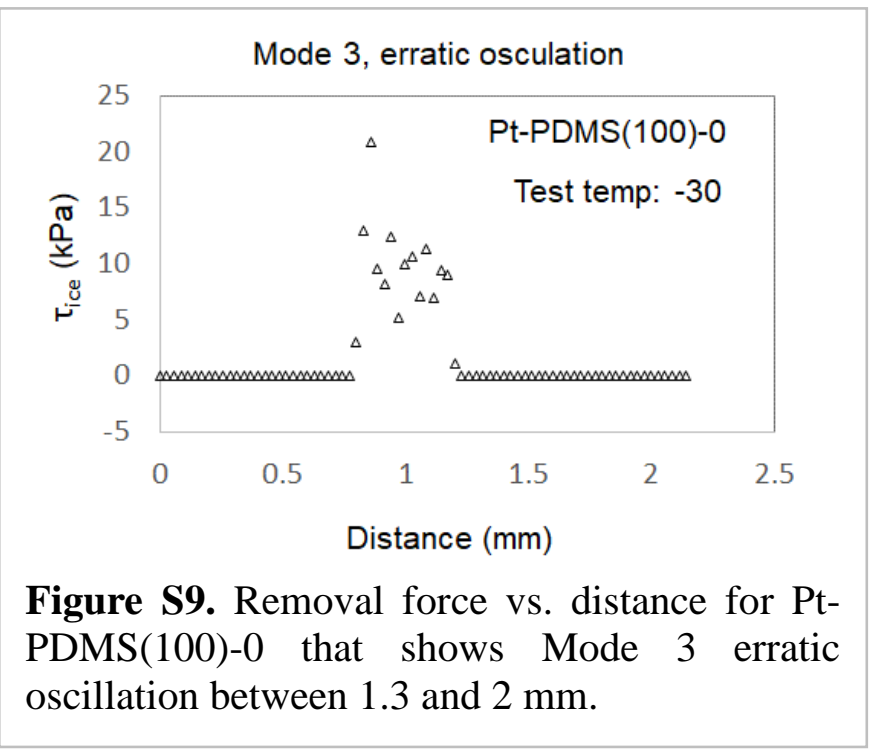




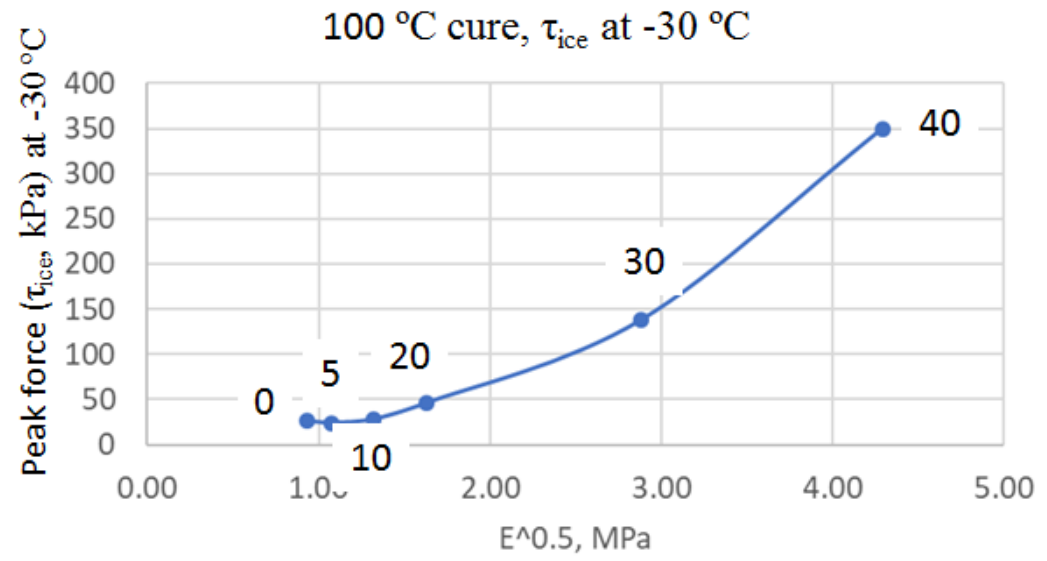

Figure S10. Peak force $\left(\tau_{\text {ice }}\right)$ versus $\left(E^{\prime}\right)^{1 / 2}$ for Pt-PDMS(100) MQ-R compositions. Data points are marked with wt\% MQ-R.

\section{References}

1. Uilk, J. M. M., A. E.; Fox, R. B.; Wynne, K. J., Hydrosilation-Cured Poly(dimethylsiloxane) Networks: Intrinsic Contact Angles via Dynamic Contact Angle Analysis. Macromolecules 2003, 36 (10), 3689-3694.

2. Wang, C.; Nair, S.; Wynne, K. J., Wilhelmy Balance Characterization Beyond Contact Angles: Differentiating Leaching from Nanosurface Reorganization and Optimizing Surface Modification. Polymer 2017, 116, 565-571. 